\title{
Impacto da vacinação contra rotavírus no Brasil
}

\section{Impact of rotavirus vaccination in Brazil}

\author{
Veronica A. Ambrosini ${ }^{1}$, Emerson Carraro*
}

\begin{abstract}
RESUMO
Modelo do estudo: Revisão sistemática da literatura.

Objetivo: Revisar a literatura sobre estudos realizados no Brasil comparando as taxas de infecção, hospitalização, mortalidade e genótipos circulantes de rotavírus durante o período pré e pós-vacinal.

Metodologia: Durante o mês de setembro de 2011 foram consultadas as bases de dados do Pubmed e Scielo sobre estudos com os descritores: rotavírus, Brasil e infecção; rotavírus, Brasil e hospitalização; rotavírus, Brasil e mortalidade; rotavírus, Brasil e genótipos.

Resultados: Os resultados demonstraram que as infecções por rotavírus continuam a causar doença significativa, apesar da grande redução nas taxas de infecção (7,4 a 77\%), hospitalização (12,3 a 55,4\%) e mortalidade (22 a 50\%). Contudo, após a introdução da vacina foi demonstrada importante alteração de genótipos circulantes de rotavírus, com substituição em diversas regiões do país do genótipo G1P8 por G2P4.

Conclusão: Após o início da vacinação contra rotavírus no Brasil houve importante redução nas taxas de infecção, hospitalização e mortalidade por diarréia, concomitante à alteração na circulação de genótipos de rotavírus, que aponta para a necessidade de monitoramento sobre uma possível atualização de novos genótipos na composição dos imunizantes como forma de garantir a continuidade de sua eficácia.
\end{abstract}

Palavras-chave: Rotavirus. Infecciones por Rotavirus. Hospitalização. Mortalidade. Genótipo. Vacinação.

\section{Introdução}

O rotavírus é um dos mais importantes agentes etiológicos da doença diarréica de modo geral. Está associado a $30-50 \%$ dos casos de diarréia grave e aproximadamente um terço das hospitalizações por diarréias. ${ }^{1}$ A doença está associada a aproximadamente 600 mil óbitos, 2 milhões de hospitalizações, 25 milhões de atendimentos de saúde e 111 milhões de episódios de diarréia em crianças jovens a cada ano no mundo todo. ${ }^{2}$ Em estudo mais recente, envolvendo 22 países da América Latina e Caribe, demonstrou que a incidência de gastroenterites para esse vírus é de 170 por 1000 crianças ao ano. ${ }^{3}$

No Brasil, ao menos 36 estudos da era prévacinal demonstraram que o rotavírus é o responsável por 4,5-44\% dos casos de diarréia aguda entre os pacientes internados ou não. ${ }^{4}$ As recentes investigações epidemiológicas do país indicam uma estimativa de 12.513 hospitalizações e 2.475 óbitos associados a este vírus. $^{5}$

A apresentação clínica clássica da infecção por
1- Farmacêutica, Discente do Curso de Pós-graduação em Ciências Farmacêuticas, Universidade Estadual do Centro-OestePR.

2- Professor Doutor do Curso de Farmácia, Universidade Estadual do Centro-Oeste-PR.
${ }^{*}$ Correspondência: Laboratório de Virologia. Rua Simeão Camargo varela de Sá, 03 CEP: 85010-080 - Vila Carli, Guarapuava -PR, Brasil. Fone/fax: 55(42)36298137 E-mail: emersoncarraro@bol.com.br.

Artigo recebido em 02/05/2012 Aprovado para publicação em 10/08/2012 
rotavírus se caracteriza pelo início abrupto dos sintomas, com vômitos e febre alta, sobrevindo diarréia profusa, aquosa e com aspecto gorduroso. Com a continuidade do quadro, instala-se a desidratação isotônica e, em condições de agravamento, decorrendo o óbito. ${ }^{6}$

O rotavírus é um vírus da família dos Reoviridae, composto de 11 segmentos de dupla fita de RNA envolvido de três camadas de proteínas. Uma destas proteínas externas corresponde ao antígeno comum aos grupos de rotavírus, localizado no componente VP6, é responsável pela classificação sorológica destes vírus. Até o momento sete grupos distintos de rotavírus foram identificados: A, B, C, D, E, F e G, ocorrendo em diversas espécies animais, sendo que somente os grupos A, B, e C são associados a doença no homem. O grupo A é o de melhor caracterização, predominando na natureza, associado a doença no homem e em diversas outras espécies animais. As porções mais externas do vírus são constituídas das proteínas VP7 e VP4, que são os principais antígenos neutralizantes do vírus. A classificação de sorotipos de rotavírus é determinada pela combinação binária entre estas duas proteínas, através do reconhecimento com anticorpos neutralizantes, sendo a VP7 determinante do sorotipo G e a VP4 do sorotipo P. Dos 14 sorotipos G (VP7) conhecidos, 10 têm sido descritos como patógenos humanos: os tipos G1 a G4 são os mais frequientemente encontrados em todo o mundo e para os quais vacinas estão sendo desenvolvidas; já os sorotipos G5, G6 e G10 eram encontrados exclusivamente como patógenos animais. ${ }^{6}$

Taxas elevadas de incidência de rotavírus nos países desenvolvidos e em desenvolvimento sustentam a hipótese de que uma vacina, mais do que melhorias na higiene e no saneamento, seja provavelmente eficaz no controle da gastroenterite por rotavírus. ${ }^{7}$ A obtenção de uma vacina eficaz e inócua tornou-se prioridade no âmbito da Organização Mundial da Saúde (OMS) para ser administrada principalmente nas crianças até os dois primeiros anos de vida, as quais quando adoecidas podem evoluir para desidratação e óbito. ${ }^{8}$

As estratégias vacinais devem considerar que a história natural das infecções por rotavírus revela que a primeira infecção ocorre, em geral, nos primeiros meses de vida e, geralmente, também apresenta maior gravidade. À medida que a criança vai sendo exposta, repetidas vezes a diferentes cepas de rotavírus a gravidade dos casos tendem a diminuir concomitante a aquisição da imunidade. ${ }^{9}$ Como a maioria dos rotavírus compartilham os principais antígenos VP6 há a possibilidade da imunização através do uso de vacinas contendo cepas isoladas de animais. No início de 2006 foi licenciada nos EUA a vacina conhecida como Rotateq ${ }^{\circledR}$ (Merck \& Co®, EUA), que compreende uma preparação pentavalente de rotavírus de origem bovino-humana. Trata-se de produto nãoreatogênico e altamente eficaz, alcançando níveis protetores de até $100 \%$ frente aos episódios diarréicos mais graves. A composição deste imunizante derivado da cepa bovina WC3 inclui antígenos dos sorotipos G1, G2, G3, G4 e P1A[8]. ${ }^{10}$ Praticamente ao mesmo tempo, foi licenciada também a vacina RotaRix ${ }^{\circledR}$ (GlaxoSmithKline, Bélgica), que compreende uma preparação monovalente de origem humana, com especificidade antigênica G1 e P[8]. ${ }^{11}$ As duas vacinas são administradas via oral para bebês em duas (RotaRix ${ }^{\circledR}$ ) ou três doses (Rotateq $\left.{ }^{\circledR}\right)$, sendo a primeira entre a $6^{a}$ e $15^{\text {a }}$ semana de vida ${ }^{12}$ e ambas têm sido consideradas seguras e eficazes na prevenção de infecções por rotavírus. ${ }^{4}$

\section{Objetivos}

Revisar estudos da literatura avaliando o impacto da vacinação contra rotavírus quanto à taxa de infecção, hospitalização, mortalidade e genótipos circulantes no Brasil.

\section{Metodologia}

Foi realizada em setembro de 2011 uma pesquisa bibliográfica nas bases de dados Pubmed e Scielo para selecionar trabalhos sobre a epidemiologia da doença e os genótipos do rotavírus no Brasil. Para isso foram utilizados os descritores: rotavírus, Brasil e infecção; rotavírus, Brasil e hospitalização; rotavírus Brasil e mortalidade; rotavírus, Brasil e genótipos (e seus correspondentes em inglês). Após a pesquisa de estudos pelos descritores acima, os trabalhos foram filtrados para a seleção de estudos originais que permitissem a comparação de dados entre períodos anterior e posterior ao início da vacinação no Brasil, em março de 2006. Contudo, permaneceram na análise estudos envolvendo a genotipagem de rotavírus somente em amostras do período pós-vacinal a fim de avaliarmos o impacto da vacinação na alteração dos genótipos circulantes.

\section{Resultados}

Foram encontrados, na base de dados Pubmed, 97 estudos relacionados aos descritores rotavírus, Bra- 
sil e infecção; 41 a partir dos descritores rotavírus, Brasil e hospitalização; 27 trabalhos relacionados aos descritores rotavírus, Brasil e mortalidade; e 98 com os descritores rotavírus, Brasil e genótipos. Após seleção dos trabalhos envolvendo a coleta de dados de pacientes antes e após o início da vacinação restaram oito estudos descrevendo o impacto nas taxas de infecção, seete sobre hospitalização e três sobre mortalidade, quanto ao impacto sobre os genótipos de rotavírus foram considerados 11 contendo informações após a introdução da vacina no Brasil. A mesma pesquisa foi realizada na base de dados Scielo, que resultou em 13 trabalhos com os descritores rotavírus, infecção e Brasil; dois envolvendo rotavírus, hospitalização e Brasil; nenhum estudo com os descritores rotavírus, Brasil e mortalidade; e cinco publicações envolvendo rotavírus, Brasil e genótipo. Após comparação das duas bases de dados foram excluídos os estudos repetidos e a Tabela 1 sumariza os 16 estudos restantes a serem discutidos.

\section{Discussão}

A presente revisão inclui vários estudos realizados no Brasil durante o período após a inclusão da vacina Rotarix no calendário nacional de imunização. Após sua aplicação inicial no Brasil, México e Venezuela sua eficácia sugerida foi de $73 \%$ para os casos graves de gastroenterites por rotavírus. ${ }^{13} \mathrm{Com}$ base nestes estudos iniciais de eficácia da vacina Rotarix estima-se que a vacinação contra rotavírus no Brasil possa prevenir anualmente 1.804 mortes, 91.127 hospitalizações e 550.198 visitas ambulatoriais, reduzindo $76 \%$ dos encargos gerais de saúde relacionados com gastroenterites por rotavírus. ${ }^{7}$ Outra estimativa aponta a capacidade de prevenir $54 \%$ dos casos de gastroenterites por rotavírus, $75 \%$ das mortes por diarréia em um período de cinco anos representando uma economia de $\mathrm{R} \$ 38.536 .514,00$ para o sistema público de saúde do Brasil. ${ }^{14}$ Um modelo econômico aplicado para oito países da América Latina estimou que a vacina de rotavírus poderia prevenir $65 \%$ das consultas médicas, mortes e custos relacionados com gastroenterites por rotavírus. ${ }^{15}$

Na presente revisão todos os estudos encontrados descrevem que após o início da prática de imunização contra rotavírus no país houve uma redução no número de infecções, hospitalizações e mortalidade em crianças. Essa redução foi variável conforme o estudo, pois nem todos envolviam somente crianças vacinadas e nem as mesmas idades. Podemos evi- denciar que a as maiores reduções de infecção foram obtidas em crianças mais jovens, menores que um ano de idade, com redução de $35,6 \%$, sendo que esse número decresce para $12,3 \%$ quando analisamos crianças entre um e quatro anos de idade. ${ }^{16}$ Dado semelhante foi descrito por Sáfadi et al ${ }^{17}$ que demonstrou uma redução de 42,2\% no número de infecções por rotavírus entre crianças menores que cinco anos de idade, entre os anos de 2004 e 2008. Contudo, as maiores reduções em números de infecção foram de $77 \%$ em crianças com até 11 meses de idades em Recife, entre 2006 e 2007, sendo encontrado também uma redução de $24 \%$ para as crianças com mais de 12 meses de idade. ${ }^{18}$

Outra variação descrita nos estudos de infecção por rotavírus ocorreu conforme a região estudada. Levantamento realizado em trinta centros de atendimento de saúde ambulatorial no estado de São Paulo demonstrou redução no número de casos positivos para rotavírus de 65,7\% em 2004 para 50\% em 2007. ${ }^{19}$ Já outro estudo realizado no Rio de Janeiro no mesmo período mostrou redução mais significativa, partindo de $38 \%$ em 2005 para $24 \%$ em $2007 .{ }^{20}$ Redução ainda maior foi descrita no estado de Sergipe, partindo de $24 \%$ em 2006 para 9,5\% e 7,4\% em 2007 e 2008, respectivamente. ${ }^{21}$

O mesmo fenômeno de impacto positivo da vacinação contra rotavírus foi encontrado quando avaliamos a hospitalização devido à diarréia. Nos anos seguintes ao início da campanha de vacinação nacional foram encontradas significativas redução nas taxas de hospitalizações por diarréia, sem considerar sua etiologia, que chegaram a diminuir em 55,4\% entre $2004 \mathrm{e}$ 2007 no Sergipe, ${ }^{21} 29 \%$ entre 2004 e 2008 em São Paulo17 e $17 \%$ em crianças com até cinco anos de idade entre 2007 e 2009 no Brasil. ${ }^{22}$ Benefícios ainda maiores foram encontrados quando avaliadas hospitalizações por gastroenterites devido ao rotavírus dentre as faixas etárias menores, como de $82,1 \%$ dentre crianças até 11 meses de vida,17 43,9\% para as crianças menores que um ano de idade, ${ }^{23}$ até $25 \%$ em crianças com menos de dois anos de idade, correspondendo ao grupo de maior cobertura vacinal,22 chegou até $94,7 \%$ em crianças abaixo de 11 meses de idade e $65,1 \%$ em crianças com idade acima de 12 meses. ${ }^{24}$

Quando avaliamos índices de mortalidade de crianças com até cinco anos de idade, encontramos um declínio de $22 \%$ logo nos três anos seguidos ao início da vacinação contra rotavírus (2007-2009). O impacto na mortalidade foi maior em crianças menores de dois anos de idade (redução de até $28 \%$ ), em 


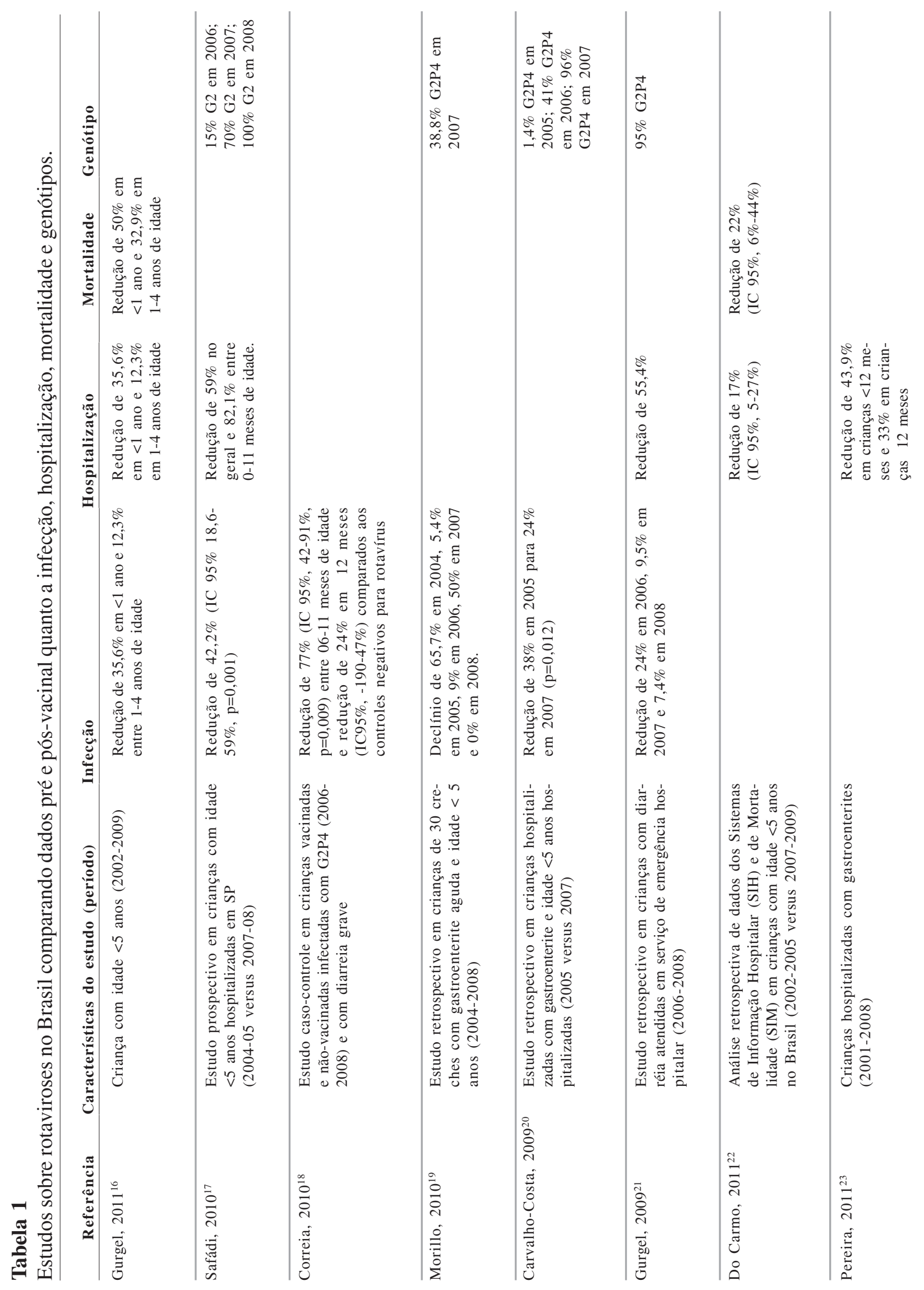




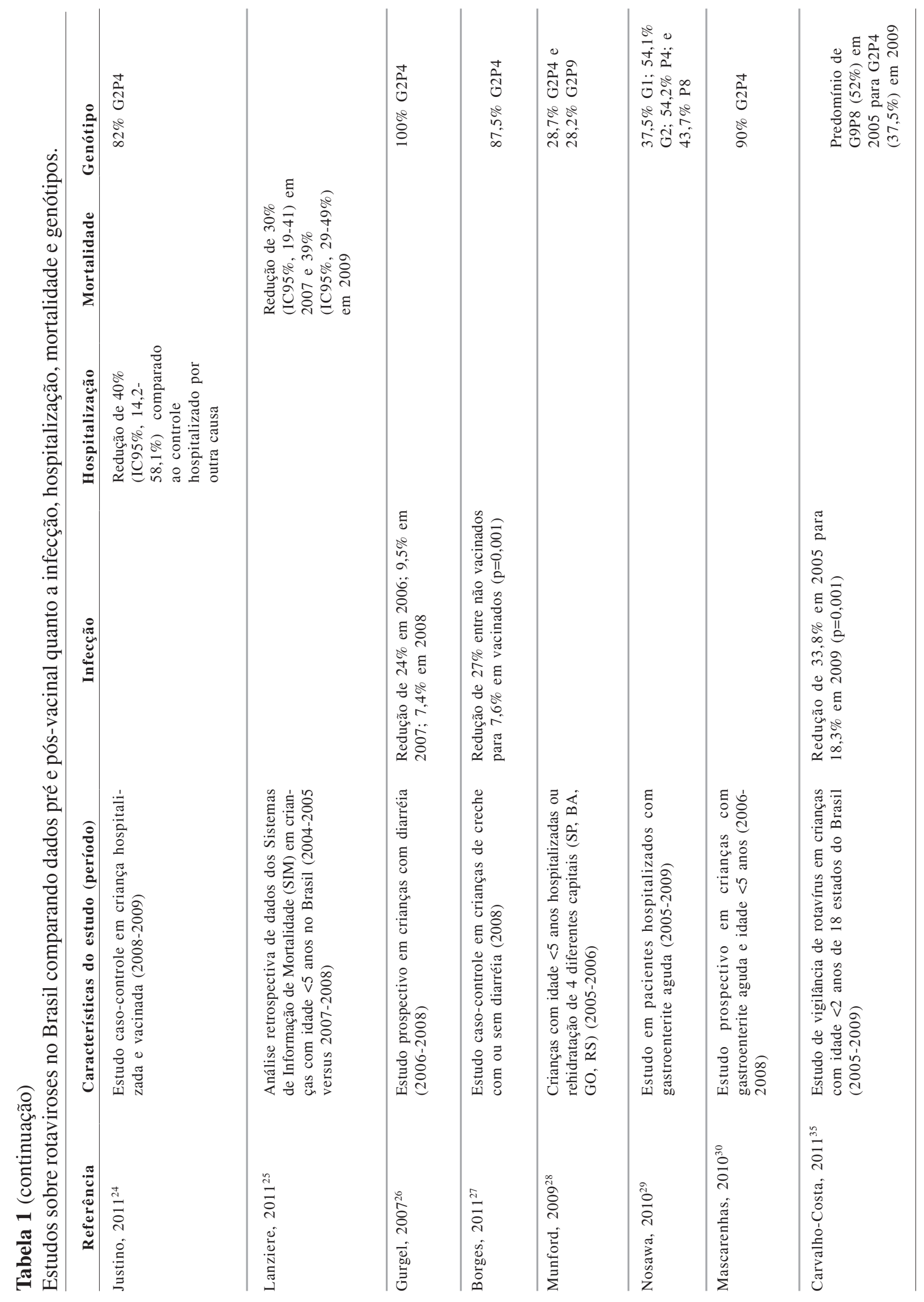


contrapartida a redução de $4 \%$ entre as crianças acima de dois anos de idade que não receberam a vacina. ${ }^{22}$ Em outro estudo, a redução no coeficiente de mortalidade chegou a 50\% em crianças menores de um ano de idade, com redução de $32,9 \%$ entre crianças de 1 e 4 anos. ${ }^{16}$ Os dados do Programa Nacional de Imunização e do Sistema de Informação de Mortalidade também confirmam esse benefício, em que a mortalidade entre crianças menores de um ano de idade decresceram em 30\% e 39\% nos anos de 2007 e 2008 , respectivamente. Contudo, a redução foi menor dentre as crianças com idade de um a cinco anos, com 29\% em 2007 e $33 \%$ em 2008. ${ }^{25}$

Logo após a introdução da vacina monovalente (G1P8) Rotarix ${ }^{\circledR}$ no calendário nacional de imunização, foram realizados estudos no nordeste que sugeriram a hipótese de que a vacina estaria selecionando o genótipo G2, já que esse genótipo teve uma maior incidência após o período de $2006 .{ }^{26}$ Apesar da vacinação resultar em redução global de diarréia, consultas e internações por rotavírus, outros autores confirmaram essa importante alteração na epidemiologia molecular dos genótipos de rotavírus circulantes no país. No Nordeste, concomitante a redução no número de casos de infecção por rotavírus foi demonstrado que o genótipo G2, encontrado em 1,4\% das amostras em 2005, passou a uma incidência de 44\% em 2006 e $95 \%$ em 2007. ${ }^{21}$ Em Goiânia, no ano de 2008 o G2 foi responsável por $62,5 \%$ das infecções por rotavírus, ${ }^{27}$ outro estudo realizado nas capitais São Paulo, Salvador, Goiânia e Porto Alegre demonstraram predomínio de G2P $4^{28}$ e em três cidades do Paraná, entre 2005 e 2009, demonstrou-se a prevalência do genótipo de G2. ${ }^{29}$ Ainda, no estado de São Paulo uma caracterização genotípica em trinta centros de atenção a saúde diários apresentou no ano de 2004 uma prevalência para os genótipos G9P8 e G1P8, em 2005 por G1P8 e no ano de 2007 prevaleceu o G2P4. ${ }^{19}$ Em um hospital sentinela em São Paulo as taxas do genótipo G2 foram 15\%, 70\% e 100\% nos anos 2006, 2007 e 2008, respectivamente. ${ }^{17}$ Já no estado do Pará esse genótipo foi prevalente (90\%) nos anos de 2006 a 2008 seguido do G1P8 6,67\% e G9P8 3,33\%. ${ }^{30}$ Da mesma forma, no Rio de Janeiro o genótipo G2 teve o perfil de predomínio ao longo dos últimos anos: 1,4\% em 2005, 44\% em 2006 e $96 \%$ em 2007. ${ }^{20}$

Buscando avaliar o histórico da variabilidade de rotavírus circulantes no país, uma recente revisão descreveu grande diversidade de genótipos no período prévacinal (1982-2005), destacando-se que o genótipo G2 apresentou grande circulação no período de 1982 até
1995 (26\%) e sofreu redução (2\%) na detecção no período seguinte, entre 1996 até 2005. ${ }^{31}$ Nos anos de 1990 a 2009 os genótipos que tiveram maior incidência em países da América Latina e Caribe foram G1, G9 e G2 com 34,2\%, 14,6\% e 14,4\% respectivamente, sendo que o G2 reemergiu e o G5 praticamente desapareceu. ${ }^{3}$ Esses dados sugerem a possibilidade de um ressurgimento natural do genótipo G2, o que tende a ocorrer em ciclos de 10 anos. ${ }^{3}$ Embora tal periodicidade possa estar relacionada à introdução da vacina, o ressurgimento do genótipo G2 provavelmente não tem associação à vacinação, como foi demonstrado em estudos realizados com crianças não vacinadas demonstrando alta incidência do genótipo G2P4 em Portugal ${ }^{32}$, Honduras ${ }^{33}$ e El Salvador. ${ }^{34}$ Ainda, outro estudo envolvendo dezoito estados do Brasil demonstrou que nos anos de 2005-2008 reemergiu o G2P4, porém, já no ano de 2009 houve um importante decréscimo, refletindo as oscilações naturais de genótipos. ${ }^{35}$

A OMS enfatiza a necessidade de conhecimento prévio da prevalência dos genótipos circulantes em vários cenários geográficos onde a vacina foi introduzida, assim como a contínua monitoração dos genótipos circulantes. $\mathrm{O}$ desafio atual das autoridades nacionais está na garantia de correlação de proteção gerada pela imunização utilizando uma vacina monovalente frente à infecção pelos demais genótipos circulantes. $^{4}$

\section{Conclusão}

As infecções por rotavírus no trato gastrointestinal continuam a causar doença significativa, apesar da prática de imunização com a vacina atual mostrar importante impacto na redução das taxas de infecção, hospitalização e mortalidade. Contudo, alterações recentes no predomínio de genótipos circulantes podem ter impacto nas vantagens do uso dessa vacina. Assim sendo, uma vigilância constante da circulação de genótipos de rotavírus em pacientes vacinados ou não é necessária para monitoramento da necessidade de atualização de novos genótipos na composição dos imunizantes como forma de garantir a continuidade de sua eficácia. Apesar de vários estudos apresentarem predomínio de G2P4 após introdução da vacina esta tem se demonstrado efetiva na redução de infecção hospitalização com tudo a possibilidade da emergência de novos sorotipos exige continua realização de estudos de vigilância de epidemiologia molecular entre crianças. 


\begin{abstract}
Study Design: Sistematic Review of literature.

Aims: In this work, national studies were reviewed in the literature by key words infection, hospitalization, mortality, and rotavirus genotypes during pre e postvaccinal periods.

Methodology: Pubmed and Scielo databases were searched for associated descriptors: rotavirus, Brazil and infection; rotavirus, Brazil and hospitalized; rotavirus, Brazil and mortality; rotavirus, Brazil and genotypes.

Results: Results appointed for significant reduction of infection (7.4-77\%), hospitalization (12.3-55.4\%), and mortality (22-50\%), besides rotavirus gastroenteritis continue to be an important disease. After introduction to immunization practices, great changes of rotavirus genotypes were described, with G2P4 predominance in substitution to G1P8.

Conclusion: The started of rotavirus vaccination results important impact of rotaviruses infection, concomitant to alteration of rotavirus genotype circulation implicating to need for molecular surveillance to change of rotavirus genotypes compounds in vaccine to efficacy guaranteed.
\end{abstract}

Key-Words: Rotavirus. Rotavirus Infections. Hospitalization. Mortality. Genotype. Vaccination.

\section{Referências Bibliográficas}

1. Parashar UD, Hummelman MG, Miller AM. Global illness and deaths caused by rotavirus disease in children. Emerg Infect Dis. 2003; 9:565-72.

2. Parashar UD, Gibson CJ, Bresee JS, Glass RI. Rotavirus and severe childhood diarrhea. Emerg Infect Dis. 2006; 12:304-6.

3. Linhares AC, Stupka JA, Ciapponi A, Bardach AE, Glujovski $D$, Aruj PK, et al. Burden and typing of rotavirus group $A$ in Latin America and the Caribbean: systematic review and meta-analysis. Rev Med Virol.. 2011;21(2):89-109.

4. CDC. Prevention of rotavirus gastroenteritis among infants and children recommendations of the Advisory Committee on Immunization Practices (ACIP). MMWR 2009; 58(RR02):1-25.

5. Constenla DO, Linhares AC, Rheingans RD, Antil LR, Waldman $E A$, da Silva LJ. Economic impact of a rotavirus vaccine in Brazil. J Health Popul Nutr. 2008; 26(4):388-96.

6. Kapikian AZ, Chanock RM. Rotaviruses in: Fieds BN, Knipe DM, Howley PM, editors. Virology. Philadelphia: LippincottRaven Publishers; 1996. p.1657-708.

7. Linhares AC, Bresee JS. Rotavirus vaccines and vaccination in Latin America. Rev Panam Salud Publica 2000; 8:30531.

8. Linhares AC, Oliveira CS, Batista NA. Reanálise da vacina tetravalente (RRV-TV) no contexto da prevenção das gastroenterites por rotavírus. Rev Pan-Amaz Saúde 2010; 1:173-9.

9. Glass RI, Bresee JS, Turcios R. Fischer TK, Parashar UD, Steele AD. Rotavirus vaccines: targeting the developing world. J Infect Dis 2005; 192(Suppl 1):S160-6.

10. Vesikari T, Matson D. O, Dennehy P, Van Damme P, Santosham $M$, Rodriguez Z, et al. Safety and efficacy of a pentavalent human-bovine (WC3) reassortant rotavirus vaccine. N. Engl. J Med. 2006, 354:23-33.

11.Bernstein DI, Sack DA, Reisinger K, Rothstein E, Ward RL. Second-year follow-up evaluation of live, attenuated human rotavirus vaccine $89-12$ in healthy infants. J Infect Dis 2002; 186:1487-9.

12. WORD HEALTH ORGANIZATION. Rotavirus Vaccines; an update. Weekly epidimiological record 2009, 84(51-52):537.
13. Perez-Schael I, Salinas B, Tomat M, Linhares AC, Guerrero ML, Ruiz-Palacios GM, et al. Efficacy of the human rotavirus vaccine RIX4414 in malnourished children. J Infect Dis 2007; 196:537-40.

14. de Soárez PC, Valentim J, Sartori AM, Novaes HM. Costeffectiveness analysis of routine rotavirus vaccination in Brazil. Rev Panam Salud Publica 2008; 23:221-30.

15. Rheingans RD, Constenla D, Antil L, Innis BL, Breuer T. Potential cost-effectiveness of vaccination for rotavirus gastroenteritis in eight Latin American and Caribbean countries. Rev Panam Salud Publica 2007; 21:205-16.

16. Gurgel RQ, Ilozue C, Correia JB, Centenari C, Oliveira SM, Cuevas LE. Impact of rotavirus vaccination on diarrhoea mortality and hospital admissions in Brazil. Trop Med Int Health 2011; 16:1180-4.

17. Sáfadi MA, Berezin EM, Munford V, Almeida FJ, de Moraes JC, Pinheiro CF, et al. Hospital-Based Surveillance to Evaluate the Impact of Rotavirus Vaccination in São Paulo, Brazil. Pediatr Infect Dis. 2010; 29:1019-22.

18. Correia JB, Patel MM, Nakagomi O, Montenegro FM, Germano EM, Correia NB, et al. Effectiveness of monovalent rotavirus vaccine (Rotarix) against severe diarrhea caused by serotypically unrelated G2P[4] strains in Brazil. J Infect Dis. 2010; 201:363-9.

19. Morillo SG, Luchs A, Cilli A, Costa FF, Carmona Rde C, Timenetsky Mdo C. Characterization of rotavirus strains from day care centers: pre- and post-rotavirus vaccine era. $J$ Pediatr. (Rio J) 2010; 86:155-8.

20. Carvalho-Costa FA, Araújo IT, Santos de Assis RM, Fialho AM, de Assis Martins CM, Bóia MN, et al. Rotavirus genotype distribution after vaccine introduction, Rio de Janeiro, Brazil. Emerg Infect Dis. 2009; 15:95-7.

21. Gurgel RG, Bohland AK, Vieira SC, Oliveira DM, Fontes PB, Barros BF, et al. Incidence of rotavirus and all-cause diarrhea in northeast Brazil following the introduction of a national vaccination program. Gastroenterology. 2009; 137:1970-5.

22. do Carmo GM, Yen C, Cortes J, Siqueira AA, de Oliveira WK, Cortez-Escalante JJ, et al. Decline in diarrhea mortality and admissions after routine childhood rotavirus immunization in Brazil: a time-series analysis. PLoS Med. 2011; 8: e1001024. 
23. Pereira LA, Raboni SM, Nogueira MB, Vidal LR, Almeida SM, Debur MC, et.al. Rotavirus infection in a tertiary hospital: laboratory diagnosis and impact of immunization on pediatric hospitalization. Braz J Infect Dis. 2011; 15:215-9.

24. Justino MC, Linhares AC, Lanzieri TM, Miranda Y, Mascarenhas $\mathrm{JD}$, Abreu E, et al. Effectiveness of the monovalent G1P[8] human rotavirus vaccine against hospitalization for severe G2P[4] rotavirus gastroenteritis in Belém, Brazil. Pediatr Infect Dis J 2011; 30:396-401.

25. Lanzieri TM, Linhares AC, Costa I, Kolhe DA, Cunha MH, Ortega-Barria $E$, et al. Impact of rotavirus vaccination on childhood deaths from diarrhea in Brazil. Int J Infect Dis 2011; 15:206-10.

26. Gurgel RQ, Cuevas LE, Vieira SC, Barros VC, Fontes PB, Salustino EF, et al. Predominance of rotavirus $P[4] G 2$ in a vaccinated population, Brazil. Emerg Infect Dis 2007; 13: 15713.

27. Borges AM, Dias e Souza M, Fiaccadori FS, Cardoso DD. Monitoring the circulation of rotavirus among children after the introduction of the Rotarix ${ }^{\mathrm{TM}}$ vaccine in Goiânia, Brazil. Mem Inst Oswaldo Cruz 2011; 106:499-501.

28. Munford V, Gilio AE, de Souza EC, Cardoso DM, Cardoso DD, Borges AM, et al. Rotavirus gastroenteritis in children in 4 regions in Brazil: a hospital-based surveillance study. J Infect Dis 2009; 200(Suppl 1):S106-13.

29. Nozawa CM, Kerntopf GF, Czernisz Eda S, Albuquerque D, Romanin P, Freitas JF, et al. Detection and characterization of human rotavirus in hospitalized patients in the cities of Ponta Grossa, Londrina and Assai - PR, Brazil. Braz J Infect Dis 2010; 14:553-7.
30. Mascarenhas JD, Lima CS, de Oliveira DS, Guerra Sde F, Maestri RP, Gabbay YB, et al. Identification of two sublineages of genotype $\mathrm{G} 2$ rotavirus among diarrheic children in Parauapebas, Southern Pará State, Brazil. J Med Virol 2010; 82:712-9.

31. Leite JPG, Carvalho C, Linhares AC. Group A rotavirus genotypes and the ongoing Brazilian experience: A review. Mem Inst Oswaldo Cruz 2008; 103:745-53.

32. Antunes H, Afonso A, Iturriza M, Martinho I, Ribeiro C, Rocha $\mathrm{S}$, et al. G2P[4] the most prevalent rotavirus genotype in 2007 winter season in an European non-vaccinated population. J Clin Virol 2009; 45:76-8.

33. Ferrera A, Quan D, Espinoza F. Increased prevalence of genotype $\mathrm{G} 2 \mathrm{P}(4)$ among hildren with rotavirus-associated gastroenteritis in Honduras. 17 European Congress of Clinical Microbiology and Infectious Diseases ICC; 2007; Munich. Hoboken (NJ): Wiley-Blackwel.

34. Patel MM, Oliveira LH, Bispo AM, Gentsch J, Parashar UD. Rotavirus P[4]G2 in a vaccinated population, Brazil. Emerg Infect Dis. 2008;14:863-5.

35. Carvalho-Costa FA, Volotão Ede M, de Assis RM, Fialho AM, de Andrade Jda S, Rocha LN, et al. Laboratory-based rotavirus surveillance during the introduction of a vaccination program, Brazil, 2005-2009. Pediatr Infect Dis J 2011; 30:S35-41. 